\title{
Traditionelle Bekleidung aus Fasern der Rüssel-Lilie (Curculigo latifolia) der Benuaq Dayak in Borneo
}

\author{
HERWIG ZAHORKA
}

\begin{abstract} Benuaq use fibres from Curculigo latifolia for textiles.

\section{Zusammenfassung} ihre Stoffe.

\section{Die Rüssel-Lilie}

\section{(Curculigo latifolia, Amaryllidaceae)}

For their warp ikat weaving the indigenous Benuaq Dayak tribe in Borneo use fibres from the leaves of Curculigo latifolia (Doyo). The weaving is carried out with a back strap loom. Doyo fibres are extremely durable and tear proof. Only the Dayak

Die indigenen Benuaq Dayak in Borneo weben ihre Ketten-Ikat-Stoffe ausschließlich mit den Fasern aus den Blättern der Rüssel-Lilie (Curculigo latifolia), Doyo genannt. Die Stoffe werden auf einem traditionellen Rückengurt-Webstuhl hergestellt. Doyo-Fasern sind äußerst haltbar und reißfest. Weltweit benutzen nur die Benuaq Dayak die Fasern von Curculigo latifolia für

Es sind etwa 20 verschiedene Curculigo-Arten bekannt, die im tropischen Amerika, Afrika, NordAustralien und im tropischen Südost-Asien heimisch sind. Die im Siedlungsgebiet der Benuaq Dayak in Ost-Kalimantan (indonesisch Borneo) in Primär- und Sekundärwäldern häufig anzutreffende Art hat knollige Rhizome. Aus diesen treiben mehrere lange, parallelnervige und spitz zulaufende Blätter aus. Pflanzen, die in der Nähe von Bächen wachsen, können mannshohe Blätter entwickeln. Sie erinnern etwas an Blätter von Orchideen oder junger Kokospalmen.

Die unscheinbaren gelben Blüten werden fast am Boden an einem kurzen, blattlosen Schaft gebildet. Der etwa $1 \mathrm{~cm}$ breite, fleischige Same ist essbar und schmeckt ein wenig nach süßlichem Gras. Um die Pflanze zu vermehren, werden reife Samen von den Pflanzen gepflückt und in die Erde gesteckt. Aber auch Rhizome werden ausgegraben, geteilt und mit etwas Erde in ein Palmblatt gewickelt, das von einem Rotangband zusammengehalten wird. Diese „Containerpflanzen" werden nach dem Austreiben in der Nähe der Langhäuser eingepflanzt.

\section{Die Gewinnung der Fasern}

Bevor die einzelnen Nerven aus den Blättern herausgezogen werden, müssen die sie umschließenden Zellstränge aufgebrochen werden. Dies geschieht mehrfach mit einem speziellen Gerät aus Bambus. Die Faserbündel werden im seich- ten Wasser eines Baches unter Wasser herausgezogen. Geschieht dies am Ufer, so werden sie sofort gründlich gewaschen, damit kein verklebender Pflanzensaft zurück bleibt, der das spätere Spleißen erschweren würde. Ein Bündel parallel zusammengelegter Fasern wird an einem Ende zusammengeknotet und zum Trocknen in die Sonne gehängt. Später werden die einzelnen Stränge dieses Bündels mit Daumen und Zeigefinger fest gerieben und dadurch aufgespleißt. Dies erfolgt Strang nach Strang von unten nach oben, was ein äußerst mühsamer Vorgang ist. Jeder Einzelstrang gibt eine riesige Zahl von feinsten Fäden frei, die ausgekämmt und versponnen werden. Dieses Spinnen erfolgt mit den Fingern durch Zusammendrehen mehrerer Einzelfäden zu einem verwebbaren Fertigfaden, der auf einer Spule aufgewickelt wird. Diese Arbeit nimmt die längste Zeit in Anspruch. Die Einzelfäden sind unzerreißbar. Sie brennen ähnlich wie Baumwolle.

\section{Färben und Weben}

Die langen Kett-Fäden für das Ikat-Gewebe werden in Rahmen gespannt und an denjenigen Stellen knotenartig abgebunden, die keine Farbe aufnehmen sollen (Ikat bedeutet Knoten). Noch vor wenigen Jahrzehnten wurden zum Färben nur Farben von einheimischen Pflanzen verwendet; nachfolgend sind einige Färbematerialien aufgelistet:

Rot: aus den Samen von Bixa orellana oder einem roten Gestein, das regional Ludu genannt wird. 
Gelb: aus den Rhizomen von Curcuma longa oder aus Codiaeum variegatum.

Grün: aus den Blättern von Jasminum sambac oder Cordyline fruticosa.

Schwarz: aus den Blättern von Archidendron spec. Indigofera tinctoria, Lieferant für die blaue Indigo-Farbe, kommt im Gebiet nicht natürlich vor und wurde auch höchstwahrscheinlich nie angebaut. Blaue Farbstoffe wurden und werden deshalb immer importiert. Heute sind es meist synthetische Farben, die meist haltbarer und farbintensiver sind.

Mit einem traditionellen Rückengurt-Webstuhl werden Doppelbahnen von 50 bis $60 \mathrm{~cm}$ Breite und 110 bis $120 \mathrm{~cm}$ Länge gewebt. Um einen Wickelrock für Frauen herzustellen, werden die Doppelbahnen an den Schmalseiten aufgeschnitten und an den Längsseiten zusammengenäht. Auch Westen für Männer und Frauen werden gewebt. Die Herstellung eines Frauenrocks dauert Monate.

\section{Die Stoffe sind reißfest und pilzresistent}

Diese Kleidungsstücke halten Generationen lang. Das Gewebe fühlt sich hart an und scheuert sich niemals durch, so als ob es aus mittelstarken Perlonfäden hergestellt worden wäre. Das Geheimnis der Dauerhaftigkeit ist wahrscheinlich die Pilzresistenz. Im Klima der sehr feuchten Tropen wird Baumwollgewebe sehr schnell morsch. Curculigo-Fasern verlieren dagegen nicht an Zug- und Scherfestigkeit. Es besteht daher die Wahrscheinlichkeit, dass Curculigo latifolia ein natürliches Fungizid in den Fasern eingelagert hat. Auch Motten gehen nicht an Gewebe aus dieser Pflanze. Curculigo-Kleider werden nur bei besonderen festlichen oder repräsentativen Anlässen getragen wie bei Trauungen, Totenfesten oder bei Empfängen besonderer Gäste. Je mehr Ikat-Bänder in eine Stoffbahn eingewebt sind, desto wertvoller ist sie.

Abb. 1(oben): Geschmückte Benuaq Dayak-Frauen bei einem Fest mit traditionellen Kleidern aus Fasern der Curculigo.

Abb. 2 (Mitte): Fruchtende Curculigo latifolia.

Abb. 3 (unten): Vermehrung von Doyo in Blattcontainern.
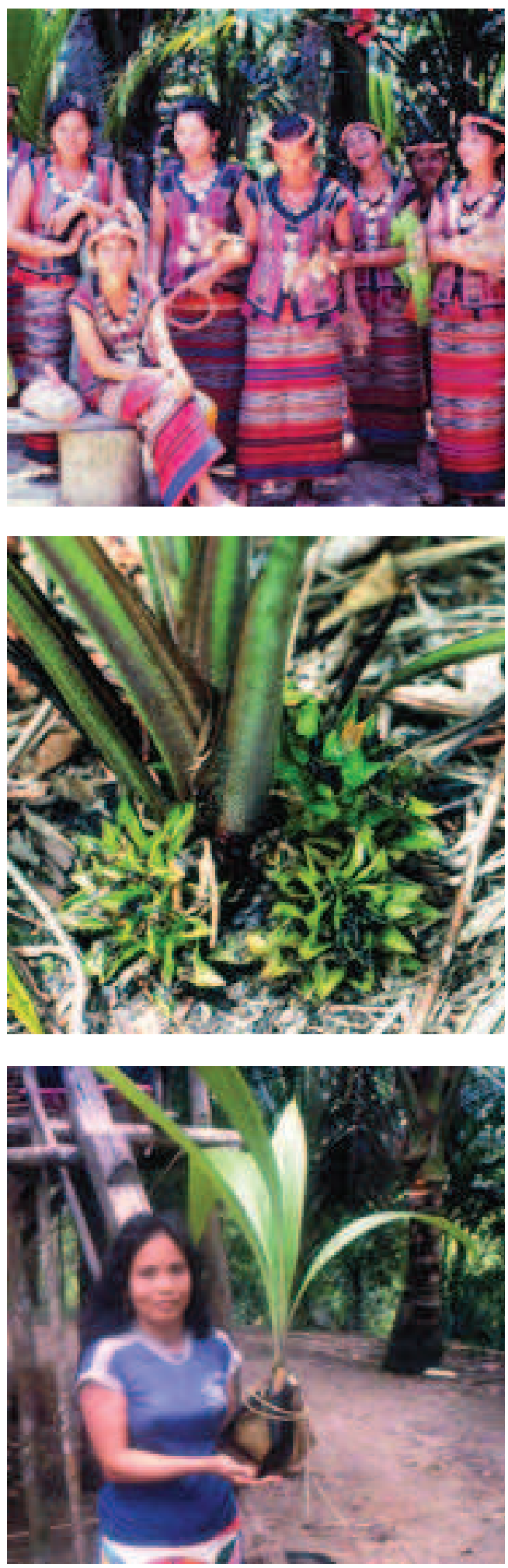

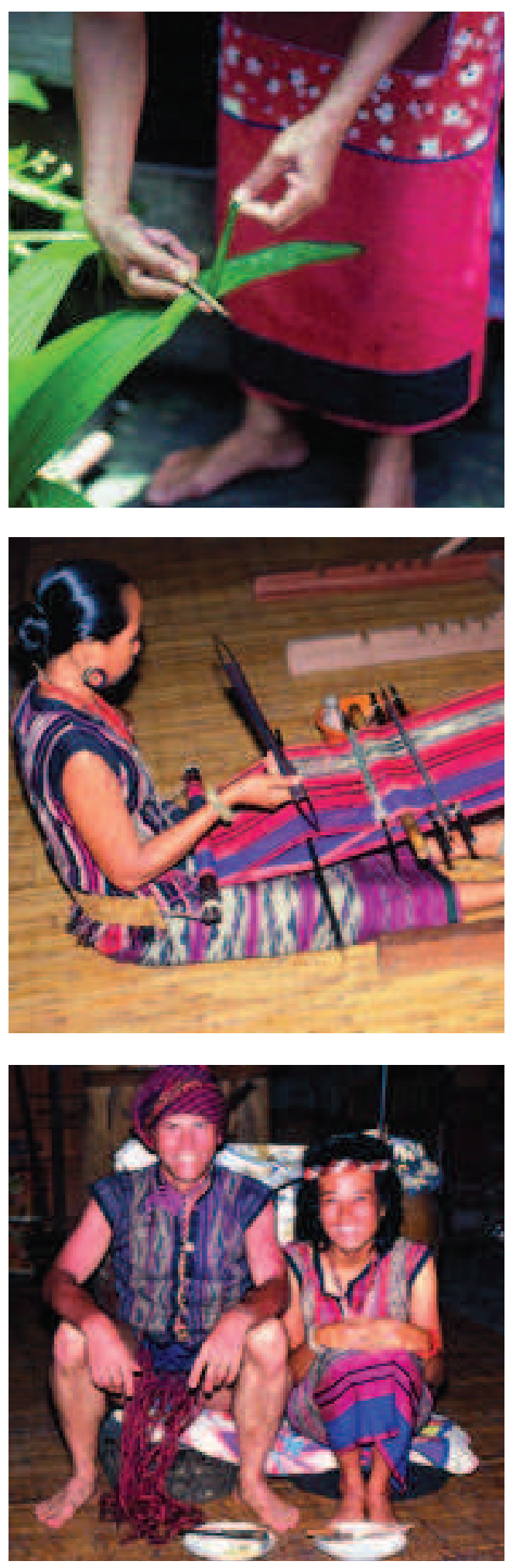

\section{Die magische Bedeutung der Ikat-Webmuster}

Die Benuaq Dayak gehen davon aus, dass ihre Umwelt von guten, bösen und wechselhaften Geistern bewohnt ist. Unter den mehr als zwanzig verschiedenen Ikat-Motiven stellen die meisten künstlerisch vereinfachte Symbole von Schutzgeistern dar. Der dargestellte Schutzgeist soll die Trägerin bzw. den Träger des Kleidungsstücks beschützen. Für den Außenseiter ist es schwierig zu erkennen, um welchen Schutzgeist es sich handelt. Aber einige Motive tragen ihre Namen, wie Naga (Drachen, kosmischer Schutzgeist), Timang (Tiger, Schutzgeist besonders bei Ritualen der Schamanen) oder Tonoi (Dorf-Schutzgeist, beseelt auch die antiken Erbstücke).

Die Juata-Wassergeister sind immer als Krokodil oder als Fisch dargestellt. Diese Geister erzeugen den Regenbogen und beschützen die Frauen, die einen Rock mit seiner Darstellung tragen, während der Menstruation, der Schwangerschaft und beim Gebären. Menschen, die die traditionellen Verhaltensregeln nicht einhalten, werden von ihnen allerdings mit Durchfall bestraft.

Eine Schlange ist das Symbol der Tonoi-DorfSchutzgeister. Schlangen sind häufige Motive an Westen und an Totenpfählen. Der Träger einer Weste mit diesen Symbolen soll von den TonoiGeistern beschützt werden. Alle alten Erbstücke der Dayak, wie Bronzegongs, antike chinesische Tonkrüge (Tempayan), mit Menschenhaar geschmückte Kopfjägerschwerter aus der Urgroßvätergeneration und Eisenholz-Blasrohre mit Giftpfeilköchern werden von Tonoi-Geistern bewacht.

Abb. 4 (oben): Aufbrechen der Faserbündel der Blattnerven. Abb. 5 (Mitte): Benuaq Dayak-Frau mit traditionellem Rückengurt-Webstuhl.

Abb. 6 (unten): Bei einem traditionellen Hochzeitsritual sitzen die mit Doyo-Kleidung ausgestatteten Brautleute auf antiken Bronzegongs. 
Nicht nur Frauen und Männer der Benuaq bekleiden sich bei besonderen Anlässen mit magischen Doyo-Kleidern. Auch Schutzgeistern gebührt eine derart würdevolle und ehrenhafte Bekleidung. Bei einem nächtlichen Heilungsritual soll der mächtige Schutzgeist Timang den Schamanen vor Angriffen böswilliger Geister schützen. Timang ist als hunde-artige Holzfigur dargestellt. Der Schamane hat sie hier sorgfältig mit einer Doyo-Stoffbahn umwickelt, um Timang zu würdigen und um ihn schutzwillig zu stimmen. Reis in den Farben Rot, Gelb, Grün, Schwarz und Weiß und entsprechend gefärbtes Wasser sollen die Geister aus allen vier Himmelsrichtungen anlocken, sie erfreuen und bei der Heilung des Kranken hilfsbereit stimmen, indem sie die Krankheit wieder zurück in ihr Geisterreich nehmen. Die hierbei verwendeten Farben werden noch immer traditionell aus den oben genannten Pflanzen gewonnen.

Abb. 7 (oben): Auch Fische symbolisieren die Juata-WasserSchutzgeister. Hier sollen sie die Frau bei Schwangerschaft und Kindsgeburt beschützen.

Abb. 8 (Mitte): Eine Weste mit vielen Ikat-Bändern, die Schlangen darstellen. Schlangen sind die symbolhafte Darstellung der Tonoi-Dorf-Schutzgeister. Der Träger soll von ihnen beschützt werden.

Abb. 9 (unten): Timang (Tiger) ist der mächtige Schutzgeist des Schamanen beim nächtlichen Krankenheilungsritual, symbolisch dargestellt als hundeartige Figur. Um Timang zu ehren und ihn willig zu stimmen, erhielt er hier eine würdige Ikat-Bekleidung aus Doyo-Fasern.
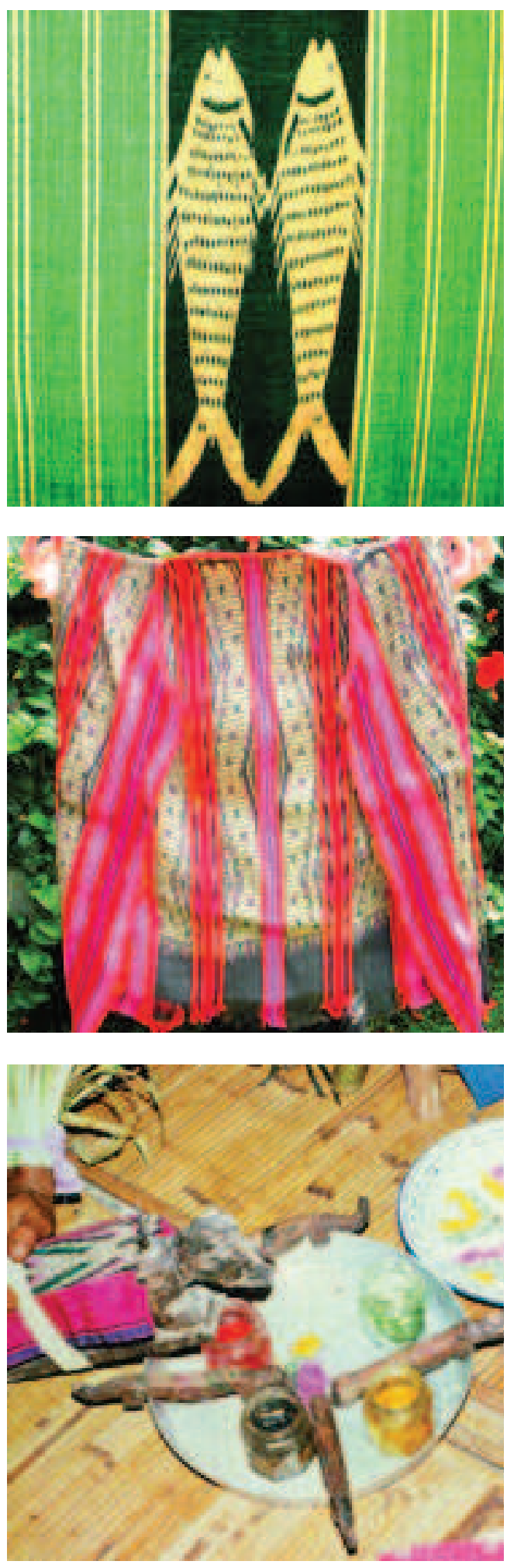